\title{
Treating bladder adenocarcinoma
}

\author{
Georgios Tsironis, Aristotle Bamias
}

Haematology-Oncology Unit, Department of Clinical Therapeutics, Alexandra Hospital, National and Kapodistrian University of Athens, Athens, Greece

Correspondence to: Georgios Tsironis. Haematology-Oncology Unit, Department of Clinical Therapeutics, Alexandra Hospital, National and Kapodistrian University of Athens, 80 Vas. Sofias Avenue, Athens 11528, Greece. Email: g_tsironis@hotmail.com.

Provenance: This is an Invited Editorial commissioned by Section Editor Xiao Li (Department of Urology, Jiangsu Cancer Hospital \& Jiangsu Institute of Cancer Research \& Nanjing Medical University Affiliated Cancer Hospital, Nanjing, China).

Comment on: Davaro F, Schaefer J, May A, et al. Invasive non-urachal adenocarcinoma of the bladder: analysis of the National Cancer Database. World J Urol 2018. [Epub ahead of print].

Submitted Oct 24, 2018. Accepted for publication Oct 28, 2018.

doi: $10.21037 /$ tau.2018.10.18

View this article at: http://dx.doi.org/10.21037/tau.2018.10.18

\section{Introduction}

Urothelial carcinoma is the sixth most common malignancy in the US with estimated 81,190 new cases and 17,240 deaths respectively for 2018. The prognosis of bladder cancer remains poor and worsens when tumor becomes muscle invasive (1). Transitional cell carcinoma is the most common bladder cancer histology, accounting for more than $90 \%$, whereas other histologies account for no more than $2-3 \%$ per variant (2). Consequently, the most robust data exist for the most common subtype, that has resulted not only into established guidelines $(3,4)$ but also to a great amount of research and to the approval of 5 new agents the previous years (5). On the other hand, adenocarcinoma of the bladder is a very rare entity, accounting for less than $2 \%$ of cases (6), with no randomized trials and no established treatment algorithms for this histology. As a result, therapeutic decisions are usually based on retrospective data. Therefore, studies such the recently published study by Facundo, Davaro et al. at World Fournal of Urology (7) are very interesting and clinically meaningful. Davaro et al. studied retrospective data from 851 pure adenocarcinoma patients from the National Cancer Database, with muscle invasive disease comparing different treatment modalities; surgery $v s$. radiotherapy $v s$. combination $v s$. no treatment (7).

\section{Prognosis}

In concordance with transitional cell carcinoma, factors that were found to be associated with worse survival for adenocarcinoma bladder cancer patients were: increased age, $\mathrm{T}$ status, lymph node involvement and metastasis. In this study, the importance of treatment centralization in high volume centers, was once again highlighted, especially for rare histologies, since, it was correlated with decreased risk for mortality.

\section{Chemotherapy}

The role of chemotherapy in the treatment of nonmetastatic muscle invasive bladder cancer of transitional cell histology is well established. Specifically in the neoadjuvant setting (8), it has been associated with a $8 \%$ benefit in 5 -year survival in a meta-analysis of 3,285 patients (9) and represents a universally accepted standard in current guidelines $(3,4)$.

On the other hand, in the absence of high-level evidence for adenocarcinomas of the bladder, the role of chemotherapy for local disease, remains debatable $(6,10)$. Neoadjuvant chemotherapy was found to decrease the frequency of non-organ-confined disease without prolonging overall survival in a subpopulation of 357 adenocarcinomas out of 2018 non-TCC bladder cancers studied retrospectively by Vetterlein et al. (11). Unfortunately, Davaro et al. reported no results regarding chemotherapy and this could be considered a clear disadvantage of the study. Chemotherapy, either perioperative or at disease relapse, could be a confounding factor of the comparison of different treatment modalities. 
In addition, this is one of the largest to date retrospective studies of purely bladder adenocarcinomas and due to this limitation, a chance for useful information regarding the role of chemotherapy in this rare bladder cancer entity has been missed (7).

\section{Cystectomy}

Radical cystectomy is the cornerstone of local TCC of the bladder, while combined chemoradiotherapy remains a valuable option for those patients that are either unfit or unwilling to undergo cystectomy $(3,4)$. The role of surgery for bladder adenocarcinomas has been evaluated with two different approaches. Firstly, Lughezzani et al. and Ghoneim et al. studied the role of cystectomy in a mixed histology population and compared its efficacy between the different histologies of bladder cancer. Adenocarcinomas were found to equally benefit from cystectomy as cancer specific mortality-free rates were not statistically different between adenocarcinomas and TCCs $(12,13)$. Davaro et al. added value to cystectomy for bladder adenocarcinomas studying the same subject from a different prospective; comparing the different treatment modalities in a pure adenocarcinoma population. At this study cystectomy was found to be the only treatment that independently contributed to a reduction in the overall mortality (7).

\section{External beam radiotherapy (EBRT)}

EBRT alone is not a preferable treatment option for bladder cancer patient as it is considered inferior to radical cystectomy (14). Nevertheless, the addition of chemotherapy improves outcomes and chemoradiation can be used as a radical treatment alternative for patients with local disease that are either unfit or unwilling to undergo cystectomy (15). Although the same strategy is intuitively followed for bladder adenocarcinomas, this is rather extrapolated from TCC guidelines and is not supported by strong evidence. Until now Zaghloul et al. had reported a possibly positive effect on the adjuvant setting through best local disease control (16). Davaro et al.'s study adds further information and questions the role of XRT to the treatment of bladder adenocarcinomas: EBRT alone was not found to offer any survival benefit and the EBRT and surgery combination lost its statistical significance when metastatic disease was excluded from the analysis (7). Interestingly, median 2 and 5 years OS in the EBRT arm was found lower than the no treatment arm.

\section{No treatment}

One of the very interesting findings of the study published in the World Fournal of Ourology is that the majority of the patients had not undergone the radical treatment that is considered the standard of care (7) and additionally some of them had not undergone any treatment at all. Although in our opinion this is mostly a result of the absence of experience as well as clear recommendations for this rare entity, another fact that may explain this attitude is the frequent poor performance status of these patients at diagnosis, which was associated with a higher incidence of 30 and 90 days' mortality. Again, missing information about chemotherapy utilization make the detailed evaluation of outcomes in this population difficult.

\section{Conclusions}

In view of the absence of level I evidence, this study provides the strongest possible evidence that radical cystectomy should be considered the standard of care for localized bladder adenocarcinomas. This modality is the only radical treatment to date, that has been proven to prolong survival in this and previous studies. The role of neoadjuvant chemotherapy and EBRT remains questionable and further data should be analyzed before these two therapies could be considered standard practice. It is of outmost importance that patients with this rare histology should be treated in high volume centers.

\section{Acknowledgements}

None.

\section{Footnote}

Conflicts of Interest: The authors have no conflicts of interest to declare.

\section{References}

1. Noone AM, Howlader N, Krapcho M, et al. SEER Cancer Statistics Review, 1975-2015, National Cancer Institute. Bethesda, MD, based on November 2017 SEER data submission, posted to the SEER web site, April 2018. Available online: https://seer.cancer.gov/csr/1975_2015/

2. Jue JS, Koru-Sengul T, Moore KJ, et al. Sociodemographic and survival disparities for histologic variants of bladder 
cancer. Can J Urol 2018;25:9179-85.

3. Chang SS, Bochner BH, Chou R, et al. Treatment of Nonmetastatic Muscle-Invasive Bladder Cancer: American Urological Association/American Society of Clinical Oncology/American Society for Radiation Oncology/ Society of Urologic Oncology Clinical Practice Guideline Summary. J Oncol Pract 2017;13:621-5.

4. Alfred Witjes J, Lebret T, Comperat EM, et al. Updated 2016 EAU Guidelines on Muscle-invasive and Metastatic Bladder Cancer. Eur Urol 2017;71:462-75.

5. Kim HS, Seo HK. Immune checkpoint inhibitors for urothelial carcinoma. Investig Clin Urol 2018;59:285-96.

6. Pons F, Orsola A, Morote J, et al. Variant forms of bladder cancer: basic considerations on treatment approaches. Curr Oncol Rep 2011;13:216-21.

7. Davaro F, Schaefer J, May A, et al. Invasive non-urachal adenocarcinoma of the bladder: analysis of the National Cancer Database. World J Urol 2018. [Epub ahead of print].

8. Bamias A, Dimopoulos MA. Neoadjuvant chemotherapy in invasive bladder cancer. Expert Rev Anticancer Ther 2005;5:993-1000

9. Yin M, Joshi M, Meijer RP, et al. Neoadjuvant Chemotherapy for Muscle-Invasive Bladder Cancer: A Systematic Review and Two-Step Meta-Analysis.

Cite this article as: Tsironis G, Bamias A. Treating bladder adenocarcinoma. Transl Androl Urol 2018;7(Suppl 6):S699-S701. doi: $10.21037 /$ tau.2018.10.18
Oncologist 2016;21:708-15.

10. Black PC, Brown GA, Dinney CP. The impact of variant histology on the outcome of bladder cancer treated with curative intent. Urol Oncol 2009;27:3-7.

11. Vetterlein MW, Wankowicz SAM, Seisen T, et al. Neoadjuvant chemotherapy prior to radical cystectomy for muscle-invasive bladder cancer with variant histology. Cancer 2017; 123:4346-55.

12. Lughezzani G, Sun M, Jeldres C, et al. Adenocarcinoma versus urothelial carcinoma of the urinary bladder: comparison between pathologic stage at radical cystectomy and cancer-specific mortality. Urology 2010;75:376-81.

13. Ghoneim MA, Abdel-Latif M, el-Mekresh M, et al. Radical cystectomy for carcinoma of the bladder: 2,720 consecutive cases 5 years later. J Urol 2008;180:121-7.

14. Shelley MD, Barber J, Wilt T, et al. Surgery versus radiotherapy for muscle invasive bladder cancer. Cochrane Database Syst Rev 2002;(1):CD002079.

15. Ploussard G, Daneshmand S, Efstathiou JA, et al. Critical analysis of bladder sparing with trimodal therapy in muscle-invasive bladder cancer: a systematic review. Eur Urol 2014;66:120-37.

16. Zaghloul MS, Nouh A, Nazmy M, et al. Long-term results of primary adenocarcinoma of the urinary bladder: a report on 192 patients. Urol Oncol 2006;24:13-20. 